\title{
浅析无人机遥感测绘技术在工程测绘中的应用
}

马霄

云南省设计院集团勘察院有限公司，云南昆明 650000

[摘要]在我国综合国力快速发展壮大的影响下，使得民众的思想意识出现了明显的变化，人们对工程建设施工质量和安全工 作越发的重视。在开展建筑工程施工工作的时候, 要想保证施工的质量, 最为重要的就是要利用有效的方法来提升工程测量 的准确性, 从而为后续的施工工作创造良好的基础。工程测量结果的准确性与建筑工程施工效果和质量密切相关。所以要想 促进建筑工程行业稳步健康发展, 我们需要切实的综合各方面情况, 来针对工程测绘技术进行不断地优化和创新, 引用最先 进的技术和理念推动建筑行业的发展。

[关键词]工程测绘; 无人机; 遥感测绘技术

DOI：10.33142/ec.v3i4.1759 中图分类号：P237;TB22 文献标识码：A

\section{Analysis on the Application of UAV Remote Sensing Surveying and Mapping Technology in Engineering Surveying and Mapping}

\author{
MA Xiao \\ Yunnan Design Institute Group Survey Institute Co., Ltd., Kunming, Yunnan, 650000, China
}

\begin{abstract}
Under the influence of the rapid development and growth of China's comprehensive national strength, the people's ideology has changed significantly, and people pay more and more attention to the quality and safety of engineering construction. When carrying out construction work, the most important thing to ensure the quality of construction is to use effective methods to improve the accuracy of engineering measurements, so as to create a good foundation for subsequent construction work. The accuracy of engineering survey results is closely related to the construction effect and quality of building engineering. Therefore, in order to promote the steady and healthy development of the construction engineering industry, we need to effectively integrate all aspects to continuously optimize and innovate the engineering surveying and mapping technology, citing the most advanced technologies and concepts to promote the development of the construction industry.
\end{abstract}

Keywords: engineering surveying and mapping; UAV; remote sensing surveying and mapping technology

引言

近年来我国社会经济水平得到了显著的提升, 在这种新的形势下, 推动了工程测绘工作的全面发展。无人机遥感 测绘技术是科学技术发展的产物, 其具备良好的优越性, 将无人机遥感测绘技术切实的引用到工程测绘工作之中, 能 够在信息收集、汇总、分析以及建模中发挥出良好的作用, 不但能够提升工作的效率, 并且对于测绘结果的准确性的 保证也是非常有帮助的。综合现实情况来说, 工程测绘中运用无人机遥感测绘技术是大势所趋, 并且已经转变成为了 当前工程测绘中的核心技术方法。

\section{1 无人机遥感测绘技术存在的优越性}

1.1 稳定性

无人机遥感技术其实质是以先进的科学技术为基础的一种操作技术, 将无人机与遥感系统充分的融合, 从而能够 实现创新的目的。其次, 在当前城市化建设工作大范围的铺展开来的带动下, 人们对建筑工工程施工质量提出了更高 的标准, 促使我们需要借助最前沿的科学技术来提升地质测量工作的整体效率和水平 ${ }^{[1]}$ 。在实际开展工程测绘工作的时 候, 切实的将无人机遥感测绘技术加以运用, 不但可以提升测绘工作的效率, 并且可以对测绘结果的准确性加以保证。 与以往陈旧性时的测量方法想对比来说, 无人机遥感测绘技术最为突出的优越性就在于准确性高, 收集数据全面, 从 而能够为工程施工质量的保证起到积极的影响作用。

1. 2 灵活性

无人机机身规格较小, 并且运用过程中十分的灵活, 不需要专门的升降场地, 所以能够被利用到各类不同的地区 测绘工作之中。近年来在科技快速发展的带动下, 无人机遥感测绘技术的整体花费得到有效的降低, 并且技术的操作 更加的方便。在实施工程测绘工作的时候, 运用污染及遥感及时需要在前期针对测绘路线进行设计, 为无人机的运行 加以规划。因为污染及遥感测绘技术具备良好的稳定性, 所以能够被运用到高强度的测绘工作之中, 并且能够对测绘 
数据的准确性加以保证。在无人机测绘完全拜托了人为驾驶的形式, 测绘效率远远的超出了航拍飞机的效率。无人机 飞行飞行高度十分灵活, 能够实现对不同情况的地点进行测绘, 保证地势测绘与航拍工作能够持续稳定的进行 ${ }^{[2]}$ 。其次, 将电子计算机技术与无人机进行融合, 测绘所获得的信息数据能够第一时间传递到检测设备中, 提升数据的利用效率。

\section{3 使用花费较少}

与航拍飞机相对比来说, 无人机控制系统操作十分简便, 并且整体成本花费只有航拍飞机的百分十二十, 工作人 员可以借助遥感系统对无人机试试操控。因为无人机的制造所使用的物料为碳纤维复合物料这类物料成本较少, 并且 后期的养护和维修工作十分简便。其次, 遥感技术所需要使用的摄像设备具备较强的综合性能, 对数据信息处理配件 的要求较低, 从而有效的实现控制数据处理花费的目的。

\section{4 分辨率较高}

在将无人机遥感技术切实的引用在工程测绘工作之中的时候, 无人机上安设的数码摄像设备往往都具有较强的准确 性, 能够从不同的角度来对测量对象进行测绘, 这样就能够有效的缓解建筑结构的遮挡问题, 增强测量工作的整体水平 ${ }^{[3]}$ 。

\section{2 无人遥感测绘技术的现状}

科学技术的快速发展, 使得各个国家都在无人机技术方面投入了更多的精力物力, 从而推动了无人机技术的稳步 发展, 使得无人机技术在社会发展方面所具有的积极影响的作用充分的施展出来。在这种形势下, 我国无人机技术也 得到了全面的发展, 并被引用到了各个领域之中, 取得了非常显著的成效。将无人机技术与遥感技术加以综合运用, 是社会发展的必然趋势, 不但可以推动社会经济的良好发展, 并且可以为国家综合国力的不断提升创造良好的基础。

\section{3 将无人机遥感技术引用到工程测绘之中}

\section{1 影像资料的获取}

在切实的运用无人机设备实施测绘工作的时候, 需要综合整个地区的实际情况对无人机的飞行路线进行合理的规 划, 并要结合天气预报情况尽可能的选择天气良好的实践进行试验飞行, 在无人机进入到飞行区域之后, 需要所有工 作人员相互配合促使无人机能够正常的非常。在实践工作中, 无人机会借助遥感技术针对测绘地区内的所有物体的坐 标进行详细的分析, 最终获得准确的定位信息。其次, 还会自行结合实际清卡㛎弓来对测绘影响的规格和各项参数进 行调整。再有, 无人机还拥有最前沿的三维模型模拟性能, 可以针对获得的坐标信息加以综合分析判断, 从而为建模 通过准确的数据, 能够保证测绘影像拥有良好的清晰度, 并且可以将整个区域内的所有较为隐蔽的地方进行全面的测 绘, 增强测绘的效果, 为后期各项工作的开展创造良好的基础 ${ }^{[4]}$ 。

\section{2 测绘数据的采集与处理}

在将无人机遥感技术加以切实运用的时候, 最为重要的工作设立完善的信息数据处理系统, 借助信息数据处理系 统可以完成对信息的高效收集和运用, 并且能够自行将各类不符合要求的信息数据加以清除, 保证测绘数据具备良好 的准确性。借助数据处理系统获取的各项信息, 测绘工作人员往往会利用有效的方法将他们存储在电子设备数据系统 之中。其次, 在实施数据处理工作的时候, 使用无人机遥感测绘技术中 GPS 系统相关技术处理软件, 能够提升数据信 息的处理效率，更好的保证数据的完整性和安全性。

\section{3 低空作业中无人机遥感技术的应用}

对于无人机起降条件来说, 其一如果在山区, 尤其是较高海拔的山区, 自然起降会存在的一定的困难; 其二如果 在平原地区, 基于恶劣天气原因下, 也会给无人机的起降增加一定的难度。为了能够在工程测绘中更好的应用, 此时 可以将无人机进行低空航拍。除此之外, 在进行无人机遥感测绘技术设计过程中, 能够涉及到应急事件等不同的范围 ${ }^{[5]}$ 。 目前, “无人飞艇低空航测系统” 已经研制成功, 同时也有了广泛的应用, 这种系统通过创新自检校, 在最大程度上减 小由于轻薄机械形变而导致的误差。

\section{4 结语}

总的来说, 无人机遥感测绘技术是科学技术发展的产物, 与其他以往陈旧模式的测绘技术相比较来说, 拥有测绘 效果强, 准确度高的优越性。将无人机遥感测绘技术切实的引用到工程测绘工作之中, 能够起到积极的影响作用, 保 证各项工作能够按照既定的计划有序的开展, 并且还可以对测绘结果的准确性加以保证。所以在工程测绘工作开展中, 施工单位管理层级人员需要对无人遥感测绘技术的重要性加以正确的认识, 并在实践中切实加以利用, 为建筑行业稳 定健康发展创造良好基础。

\section{[参考文献]}

[1]农堂起. 工程测绘中无人机遥感测绘技术的应用 [J]. 科技创新与应用, 2020 (08) : 172-173.

$[2]$ 白玉灵.浅谈无人机遥感测绘技术在工程测绘中的应用 $[\mathrm{J}]$. 科技风, 2020 (02) : 7.

[3] 张博.无人机遥感技术在工程测绘中的应用探讨 [J].住宅与房地产,2019(34): 203.

[4] 黎信宏, 吴侠.浅析无人机遥感测绘技术在工程测绘中的应用 [J].世界有色金属, 2019(17): 297-298.

[5]张洁.无人机遥感测绘技术在工程测绘中的应用探究 [J].信息与电脑 (理论版), 2019 (15) : 13-15.

作者简介: 马霄 (1985.7-), 男, 中国地质大学 (武汉), 测绘工程, 云南省设计院集团勘察院有限公司, 员工, 工程师。 